\title{
EXTRAPOLATION TECHNIQUES AND THE COLLOCATION METHOD FOR A CLASS OF BOUNDARY INTEGRAL EQUATIONS
}

\author{
RICARDO CELORRIO' ${ }^{1}$ and FRANCISCO-JAVIER SAYAS ${ }^{2}$
}

(Received 31 October 1997; revised 19 February 1999)

\begin{abstract}
In this paper we prove the existence of asymptotic expansions of the error of the spline collocation method applied to Fredholm integral equations of the first kind with logarithmic kernels. These expansions justify the use of Richardson extrapolation for the acceleration of convergence of the method. The results are stated and proven for a single equation, corresponding to the parameterization of a boundary integral equation on a smooth closed curve. As a byproduct we obtain the nodal superconvergence of the scheme. These results are then extended to smooth open arcs and to systems of integral equations. Finally we prove that such expansions also exist for the Sloan iteration of the numerical solution.
\end{abstract}

\section{Introduction}

In this paper we consider the numerical solution of some boundary integral equations (BIE). Boundary integral methods provide a useful tool for dealing with linear homogeneous boundary value problems (BVP) by means of a representation formula for the set of solutions of the differential equation plus an integral equation. In this way the dimension of the problem is reduced by one (thus, plane problems are converted into 1-dimensional integral equations) and we are allowed to deal with BVP in some unbounded domains.

The general BIE we analyse here may be written in the form

$$
\int_{0}^{1}\left(A(\cdot, s) \log \left(\sin ^{2}(\pi(\cdot-s))\right)+B(\cdot, s)\right) g(s) d s=f
$$

\footnotetext{
${ }^{1}$ Dep. Matemática Aplicada - E.U.I.T.I.Z., Universidad de Zaragoza - Corona de Aragón, 50009 Zaragoza, Spain; e-mail: celorrio@posta.unizar.es.

${ }^{2}$ Dep. Matemática Aplicada - Centro Politécnico Superior, Universidad de Zaragoza - María de Luna, 3, 50015 Zaragoza, Spain; e-mail: jsayas@posta.unizar.es.

(9) Australian Mathematical Society 2001, Serial-fee code 0334-2700/01
} 
This equation actually corresponds to the parameterization of a BIE. We have thus transformed a problem on a curve into a periodic problem on the real line.

Different Galerkin schemes (with piecewise polynomials, including smoothest splines, with trigonometric polynomials, etc.) for a wide class of periodic integral equations have been analysed in the literature. Moreover, their properties are not only well understood but also good enough from the point of view of the order of convergence. However, collocation-type methods are those most used in the engineering world, because of the simplicity of implementation and of the clarity of the ideas involved.

The analysis of collocation methods for logarithmic integral equations such as (1) follows from a series of papers in the mid and late eighties, [1] and [11] marking the most complete contributions. In more recent times, this work has been extended to the integral equations arising from problems on open arcs and domains with corners, although much is still undone, especially as regards extending these results to problems on surfaces.

One important aspect of the analysis of any numerical method is the existence of an asymptotic expansion of the error. If we are approximating an unknown quantity or function $T_{0}$ by $T(h)$, depending on a unique discretization parameter $h \rightarrow 0^{+}$, an asymptotic expansion of the error (AEE in the sequel) is an expression of the form

$$
T(h)=T_{0}+T_{p} h^{p}+T_{p+1} h^{p+1}+\cdots,
$$

where the series must be understood in a formal sense (that is, limited to a finite number of terms plus a remainder) and in some norm. The applicability of (2) is twofold. On one hand, we can use the solutions in two different discretization levels, say $h$ and $h^{\prime}$, to obtain by a simple linear combination an approximation of the principal part of the error, namely $T_{p} h^{p}$. On the other hand, using several solutions (for $h_{1}, h_{2}, h_{3}, \ldots$ ) we can cancel the first terms of the AEE and hence obtain a numerical approximation of higher order. This procedure for the acceleration of convergence is often called Richardson extrapolation.

In this paper we consider spline collocation methods for (1) and prove the existence of several kinds of AEE. A precedent of this can be found in [12], where in the terminology of pseudodifferential equations, error expansions are proven to exist not for the solution itself but for the action of a smoothing functional applied to it. In the terminology of $\mathrm{BIE}$, this means for instance that we obtain pointwise AEE for the solution of the associated BVP via the representation formula on the boundary.

With very different techniques and with the logarithm playing a significant role, we prove the existence of expansions of the form.

$$
g_{h}=Q_{h} g+h^{p} Q_{h} g_{p}+h^{p+1} Q_{h} g_{p+1}+\cdots,
$$


where $g$ is the solution of (1), $g_{h}$ is its approximation by the spline collocation method and $Q_{h}$ is the interpolation operator associated with the spline space. Similar expansions for the spline Galerkin method appear in [6]. From this we are able to derive, for instance, AEE for the potentials uniformly on compact sets and also for the Sloan iteration of the solution [8].

The structure of the paper is as follows. In Sections 2 and 3 we introduce the equation of intersect and the collocation method. Sections 4,5 and 6 are devoted to proving the existence of expansions in the form of (3). These expansions are stated in Section 4 and proven in the following sections. As a consequence we prove a new nodal superconvergence result. Part of the analysis is reduced to the proof of a non-standard Euler-Maclaurin formula for functions with a logarithmic singularity. This is left for the Appendix.

A particular case is examined in Section 7, namely when coefficients and data in (1) are even functions of all their variables. This corresponds to integral equations on open curves after the cosine change of variable [19].

Finally in Section 8, we extend these results to systems of BIE with some side conditions and prove how (3) transfers into proper AEE of some postprocesses applied to the numerical solution.

NOTATION. Throughout the paper we will deal with spaces of complex-valued functions of one or two real variables, which are 1-periodic in each variable. We will denote by $\mathscr{C}^{k}$ the space of $k$ times continuously differentiable functions, with the above periodicity properties. We will not indicate whether a function is defined on $\mathbf{R}$ or $\mathbf{R}^{2}$, since such effect will be clear from the context. As usual we define $\mathscr{C}^{\infty}:=\bigcap_{k} \mathscr{C}^{k}$

\section{The integral equation}

We are interested in the numerical solution of

$$
\int_{0}^{1}\left(A(\sigma, s) \log \left(\sin ^{2}(\pi(\sigma-s))\right)+B(\sigma, s)\right) g(s) d s=f(\sigma), \quad \forall \sigma,
$$

where $A, B: \mathbf{R}^{2} \rightarrow \mathrm{C}$ are smooth 1-periodic functions, say $A, B \in \mathscr{C}^{\infty}$, and $f: \mathbf{R} \rightarrow \mathbf{C}$ is 1-periodic and continuous. We assume that

$$
A(\sigma, \sigma) \neq 0, \quad \forall \sigma \in \mathbf{R} .
$$

EXAMPLE 1. Let $\Gamma$ be a smooth simple Jordan curve parameterized by means of a $\mathscr{C}^{\infty}$ 1-periodic function $\mathbf{x}: \mathbf{R} \rightarrow \Gamma \subset \mathbf{R}^{2}$ such that $\left|\mathbf{x}^{\prime}(s)\right| \neq 0$, for all $s$ and 
$\mathbf{x}(\sigma) \neq \mathbf{x}(s)$, for $0<|\sigma-s|<1$. Consider the Dirichlet problem for Laplace's equation

$$
\left\{\begin{array}{l}
\Delta u=0, \quad \text { in } \mathbf{R}^{2} \backslash \Gamma, \\
\left.u\right|_{\Gamma}=u_{0}
\end{array}\right.
$$

and the single-layer model associated with the problem

$$
u(y):=-\frac{1}{4 \pi} \int_{\Gamma} \log |y-z|^{2} q(z) d \gamma(z), \quad y \notin \Gamma .
$$

By the continuity of the single-layer across the boundary and parameterizing the integral on $\Gamma$ we obtain an equation like (4) where

$$
\begin{aligned}
& g(s):=-\frac{1}{4 \pi} q(\mathrm{x}(s))\left|\mathrm{x}^{\prime}(s)\right|, \quad f(\sigma):=u_{0}(\mathrm{x}(\sigma)), \\
& A(\sigma, s):=1, \quad B(\sigma, s):=\log \left(\frac{|\mathrm{x}(\sigma)-\mathrm{x}(s)|^{2}}{\sin ^{2}(\pi(\sigma-s))}\right) .
\end{aligned}
$$

This equation is uniquely solvable provided that the logarithmic capacity of the curve differs from 1 (see [17] for example).

EXAMPLE 2. Let $\Gamma$ and $\mathbf{x}$ be as in the previous example. Consider the Dirichlet problem for the Helmholtz equation

$$
\left\{\begin{array}{l}
\Delta u+\lambda^{2} u=0, \quad \text { in } \mathbf{R}^{2} \backslash \Gamma, \\
\left.u\right|_{\Gamma}=u_{0},
\end{array}\right.
$$

where $\lambda \neq 0, \operatorname{Im} \lambda \geq 0$. Assume also that $-\lambda^{2}$ is not an eigenvalue of the Laplacian in the interior of $\Gamma$. Let $H_{0}^{(1)}: \mathbf{C} \backslash\left({ }_{l} \mathbf{R}^{-}\right) \rightarrow \mathbf{C}$ (with $\mathbf{R}^{-}:=\{r \in \mathbf{R}: r \leq 0\}$ ) be the Hankel function of the first kind and order 0 . Note that $H_{0}^{(1)}$ can be decomposed as

$$
H_{0}^{(1)}(z)=J_{0}(z)+\imath \frac{2}{\pi} J_{0}(z) \log z+\imath a\left(z^{2}\right),
$$

where $a$ is an entire function, $J_{0}$ is the Bessel function of order 0 and $\log$ denotes the holomorphic determination of the logarithm in $C \backslash\left(\imath \mathbf{R}^{-}\right)$which extends the real logarithm. The single layer

$$
u(\mathbf{y}):=\frac{\imath}{4} \int_{\Gamma} H_{0}^{(1)}(\lambda|\mathbf{y}-\mathbf{z}|) q(\mathbf{z}) d \gamma(\mathbf{z}), \quad \mathbf{y} \notin \Gamma,
$$


gives a solution of the Helmholtz equation satisfying Sommerfeld's radiation conditions at infinity (see [4] for instance). When taking the limit as $\mathbf{y} \rightarrow \Gamma$ and parameterizing, the resulting BIE is

$$
\frac{l}{4} \int_{0}^{1} H_{0}^{(1)}(\lambda|\mathbf{x}(\sigma)-\mathbf{x}(s)|) g(s) d s=f(\sigma),
$$

where we define $g:=q(\mathbf{x}(\cdot))\left|\mathbf{x}^{\prime}(\cdot)\right|$ and $f:=u_{0}(\mathbf{x}(\cdot))$. Because of $(7)$, the previous equation can be easily written in the form of (4).

The usual functional frame where equations like (4) are studied is that of periodic Sobolev spaces. For $r \in \mathbf{R}$ we consider the norm

$$
\|u\|_{r}:=\left(|\hat{u}(0)|^{2}+\sum_{0 \neq k \in Z}|2 k \pi|^{2 r}|\hat{u}(k)|^{2}\right)^{1 / 2},
$$

where

$$
\hat{u}(k):=\int_{0}^{1} u(s) e^{-2 k \pi i s} d s
$$

are the usual Fourier coefficients. The completion of the space of 1-periodic infinitely differentiable complex-valued functions with the previous norm is denoted by $\boldsymbol{H}^{r}$. For all $r \in \mathbf{R}, H^{r}$ is a Hilbert space whose inner product will be denoted by $(\cdot, \cdot)_{r}$. The space $H^{0}$ can be identified with $L^{2}(0,1)$. Moreover, the inner product

$$
(u, v)_{0}=\int_{0}^{1} u(s) \overline{v(s)} d s
$$

can be extended to represent the duality between $H^{r}$ and $H^{-r}$ for all $r$. Note that the spaces $H^{r}$ can also be understood as spaces of periodic distributions [20].

We denote

$$
V(\sigma, s):=A(\sigma, s) \log \left(\sin ^{2}(\pi(\sigma-s))\right)+B(\sigma, s) .
$$

Then the integral operator

$$
V g:=\int_{0}^{1} V(\cdot, s) g(s) d s
$$

can be extended to a continuous map from $H^{r}$ to $H^{r+1}$ for all $r$. Moreover, by simple properties of logarithmic operators, it can be easily verified that $V: H^{r} \rightarrow H^{r+1}$ is Fredholm of index 0 , that is, bijectivity follows from injectivity.

HYPOTHESIS 1. We assume that $V: H^{r} \rightarrow H^{r+1}$ is an isomorphism for all $r$. 
Obviously this is equivalent to demanding that $V$ is one-to-one for all $r$. Moreover, given the special structure of the kernel, this is equivalent to injectivity for any single value of $r$. If the previous hypothesis does not hold, one can always extend the equation to a uniquely solvable system by the addition of a finite number of linear integral restrictions and complex unknowns (see [4] and [13]). These systems are included in those treated in Section 7.

We then consider the operator equation

$$
V g=f .
$$

Since we are going to apply a collocation method, we assume that $f \in H^{r}$ for $r>1 / 2$ and is thus continuous by the Sobolev embedding theorem.

\section{The collocation method}

In this section we introduce the spline collocation method on a uniform mesh. Given a positive integer $N$ we define the discretization parameter $h:=1 / N$; a uniform mesh $s_{i}, i \in \mathbf{Z}$ (that is, $s_{i}-s_{j}=(i-j) h$ for all $\left.i, j\right)$; and a partition of the real line where the midpoints of the intervals are the points of the mesh $l_{i}:=\left(s_{i}-h / 2, s_{i}+h / 2\right)$, $i \in \mathbf{Z}$.

Let $m \geq 0$ be an integer and let us consider the space $V_{h}$ of the smoothest periodic splines of degree $m$ with respect to the partition $\left\{I_{j}\right\}_{j \in Z}$. That is, if $m=0$ we have the space of 1-periodic piecewise constant functions

$$
V_{h}:=\left\{u_{h} \in H^{0}:\left.u_{h}\right|_{I_{i}} \in \mathbf{P}_{0}, \forall i\right\}
$$

and for $m \geq 1$

$$
V_{h}:=\left\{u_{h} \in \mathscr{C}^{m-1}:\left.u_{h}\right|_{I_{i}} \in \mathbf{P}_{m}, \forall i\right\}
$$

where $\mathbf{P}_{m}$ is the space of polynomials with complex coefficients of degree less than or equal to $m$. The points $\left\{s_{i}+h / 2\right\}$ are often referred to as knots of the splines. Obviously $V_{h}$ is a subspace of $H^{m}$.

For $m$ even, we consider the points $z_{i}:=s_{i}$, whereas for $m$ odd we denote $z_{i}:=$ $s_{i}-h / 2$. For $u \in \mathscr{C}^{0}$ there exists a unique $Q_{h} u \in V_{h}$ such that

$$
Q_{h} u\left(z_{i}\right)=u\left(z_{i}\right), \quad \forall i,
$$

by the Schoenberg-Whitney theorem (see [7]). Since the points $\left\{z_{i}\right\}$ are taken as interpolation nodes, we will call them nodes.

The spline collocation method for (9) is the discrete problem

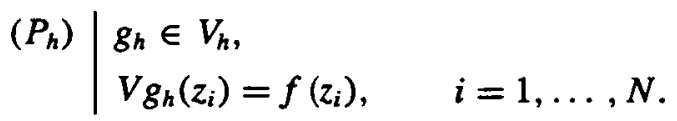


Note that we could equivalently demand that $Q_{h} V g_{h}=Q_{h} f$. In [1] it is shown that $\left(P_{h}\right)$ is uniquely solvable for $h$ sufficiently small. Then we can define the collocation operator $C_{h}: H^{r} \rightarrow V_{h}$, for $r>-1 / 2$, as the operator mapping $g$ to the unique solution of

$$
\mid \begin{aligned}
& C_{h} g \in V_{h}, \\
& Q_{h} V C_{h} g=Q_{h} V g .
\end{aligned}
$$

Stability and convergence of the method follow from [1]: for $s \in[-1, m+1 / 2)$ and $s \leq t \leq m+1, t>-1 / 2$, there exists $C=C(s, t)$ such that for $h$ small enough

$$
\left\|C_{h} g-g\right\|_{s} \leq C h^{t-s}\|g\|_{t}
$$

for all $g \in H^{t}$. Consequently the operators $C_{h}: H^{0} \rightarrow H^{0}$ are uniformly bounded for $h \leq h_{0}$. Moreover, in [11] the following optimal convergence result in weak norms is proven: for $m$ even and $g \in H^{m+2}$,

$$
\left\|C_{h} g-g\right\|_{-2} \leq C h^{m+3}\|g\|_{m+2} .
$$

\section{Asymptotic expansion of the error}

We will use the Landau symbol $\mathscr{O}$ : given $f(h ; \cdot)$ depending on the discrete parameter $h$ or on a continuous version of it (with the common feature that $h \rightarrow 0^{+}$), we write $f(h ; \cdot)=\mathscr{O}\left(h^{k}\right)$ if $h^{-k} f(h ; \cdot)$ remains bounded as $h \rightarrow 0^{+}$. Uniformity in the other variables in $f$ is not assumed unless explicitly stated. We will also use the modified symbol $\sigma_{*}$ : given a vector $\mathbf{a}_{h}=\left(a_{1}, \ldots, a_{N}\right)^{\top} \in \mathbf{R}^{N}(h=1 / N)$ we denote $a_{i}=\mathscr{O}_{*}\left(h^{k}\right)$ if $\max _{1 \leq i \leq N}\left|a_{i}\right|=\mathscr{O}\left(h^{k}\right)$.

We also denote

$$
p(m):= \begin{cases}m+2, & \text { if } m \text { is even, } \\ m+1, & \text { if } m \text { is odd. }\end{cases}
$$

As a first step we formulate an error expansion, whose proof will be given in the next section. This expansion is essentially a consistency result, the presence of the operator $C_{h}$ under the integral operator $V$ not being necessary.

PROPOSITION 1. Let $g \in \mathscr{C}^{\infty}$ and $\zeta \in[-1 / 2,1 / 2)$. Then there exists a sequence of functions $\left\{e_{k, \zeta}\right\} \subset \mathscr{C}^{\infty}$ such that for all $M$

$$
V\left(I-Q_{h}\right) g\left(s_{i}+\zeta h\right)=\sum_{k=p(m)}^{M} h^{k} e_{k, \zeta}\left(s_{i}+\zeta h\right)+\mathscr{O}_{*}\left(h^{M+1}\right)
$$


We now reformulate the discrete problem as a Petrov-Galerkin expression by the use of a set of Dirac delta distributions. For $i=1, \ldots, N$ and for $r<-1 / 2$, let $\delta_{i} \in H^{r}$ be given by the relation

$$
\left(\delta_{i}, u\right)_{0}=u\left(z_{i}\right), \quad \forall u \in \mathscr{C}^{\infty} .
$$

Consider then the discrete $N$-dimensional space $T_{h}:=\mathbf{C}\left\langle\delta_{1}, \ldots, \delta_{N}\right\rangle$. Then $\left(P_{h}\right)$ is equivalent to the problem

$$
\mid \begin{aligned}
& g_{h} \in V_{h}, \\
& \left(V g_{h}, t_{h}\right)_{0}=\left(f, t_{h}\right)_{0}, \quad \forall t_{h} \in T_{h} .
\end{aligned}
$$

The following result, proven in Section 5, is a consequence of the stability of the method.

PROPOSITION 2. There exist $\beta>0$ and $h_{0}>0$ such that for all $h \leq h_{0}, v_{h} \in V_{h}$, there exists $0 \neq t_{h} \in T_{h}$ such that

$$
\left|\left(V v_{h}, t_{h}\right)_{0}\right| \geq \beta\left\|v_{h}\right\|_{0}\left\|t_{h}\right\|_{-1} .
$$

We then obtain a kind of asymptotic expansion of the error of the collocation method.

THEOREM 3. Let $g \in \mathscr{C}^{\infty}$. Then there exists a unique sequence of functions $\left\{f_{k}\right\} \subset \mathscr{C}^{\infty}$ such that for all $M$

$$
C_{h} g-Q_{h} g-\sum_{k=p(m)}^{M} h^{k} Q_{h} f_{k}=\mathscr{O}\left(h^{M+1}\right)
$$

uniformly in R. Moreover, the bound also holds for derivatives up to order $m$.

PROOF. Take $\zeta=0$ if $m$ is even and $\zeta=-1 / 2$ if $m$ is odd in Proposition 1 and denote $g_{k}:=V^{-1} e_{k, \zeta}$. By Lemma 9, there exists a constant $C$ independent of $h$ such that for all $t_{h}=\sum_{j=1}^{N} t_{j} \delta_{j} \in T_{h}$,

$$
h \sum_{j=1}^{N}\left|t_{j}\right| \leq C\left\|t_{h}\right\|_{-1} .
$$

Then, by Proposition 1, it follows readily that for all $t_{h} \in T_{h}$

$$
\left(V g-V Q_{h} g, t_{h}\right)_{0}=\sum_{k=p(m)}^{M} h^{k}\left(V C_{h} g_{k}, t_{h}\right)_{0}+\mathscr{O}\left(h^{M+1}\right)\left\|t_{h}\right\|_{-1}
$$


To obtain (15) we have to take an additional term in Proposition 1 which tends to the remainder by the continuity of $V$ and the stability of $C_{h}$.

From here on, the proof follows exactly as the proof of Theorem 2 in [6], by simple applications of expansions (15), of Proposition 2 and of bounds of different norms in the spline spaces. Uniqueness follows from the convergence of the interpolation operator.

REMARK 4. If $g \in H^{n+1}$ and $A, B \in \mathscr{C}^{n+3}$, then there exists an asymptotic expansion like (14) up to the term $M=n-m-3$. The smoothness of the functions in the expansion is now limited. It can be proven that $f_{k} \in \mathscr{C}^{n-k}$ for all $k$.

COROLLARY 5. For $m$ even and $g$ smooth enough we have

$$
\max _{1 \leq i \leq N}\left|g\left(z_{i}\right)-C_{h} g\left(z_{i}\right)\right|=\mathscr{O}\left(h^{m+2}\right),
$$

that is, a superconvergence phenomenon in the midpoints of the mesh.

\section{Spline interpolation and the logarithmic operator}

In this section we are going to prove Proposition 1. The proof will be done in several steps. For fixed $\zeta \in \mathbf{R}$, we are interested in obtaining an expansion of $V\left(I-Q_{h}\right) g$ in the points $s_{i}+\zeta h, i \in \mathbf{Z}$. Notice that by periodicity, $\zeta$ can be taken in $[-1 / 2,1 / 2)$ and $i$ varies only in $\{1, \ldots, N\}$. From [14, Section 5] we have a local expansion of the interpolate

$$
g-\left.Q_{h} g\right|_{I_{i}}=\sum_{k=m+1}^{M} h^{k} P_{k}\left(\frac{-s_{i}}{h}\right) g^{(k)}+O_{*}\left(h^{M+1}\right)
$$

uniformly in $I_{i}$, where $P_{k}$ are fixed real polynomials of degree exactly $k$ and such that $P_{k}(-t)=(-1)^{k} P_{k}(t)$. This expansion is valid for all $g \in \mathscr{C}^{M+1}$. We denote

$$
\alpha_{k}:=\int_{-1 / 2}^{1 / 2} P_{k}(t) d t
$$

Obviously $\alpha_{k}=0$ if $k$ is odd.

For simplicity, as in the statement of Proposition 1, we will assume that $g \in \mathscr{C}^{\infty}$. We also consider the splitting $V=V_{0}+V_{1}$ where

$$
V_{0} g:=\int_{0}^{1} A(\cdot, s) \log \left(\sin ^{2}(\pi(\cdot-s))\right) g(s) d s, \quad V_{1} g:=\int_{0}^{1} B(\cdot, s) g(s) d s .
$$


Let us define the functions

$$
\beta_{k}(\sigma ; h):=\sum_{j=1}^{N} \int_{l_{j}} P_{k}\left(\frac{s-s_{j}}{h}\right) A(\sigma, s) \log \left(\sin ^{2}(\pi(\sigma-s))\right) g^{(k)}(s) d s .
$$

PROPOSITION 6. For all integer $M>m$,

$$
V\left(g-Q_{h} g\right)(\sigma)=\sum_{k=m+1}^{M} h^{k}\left(\beta_{k}(\sigma ; h)+\alpha_{k} V_{1} g^{(k)}(\sigma)\right)+\sigma\left(h^{M+1}\right)
$$

uniformly in $\sigma \in \mathbf{R}$

PROOF. Consider the function $p_{h} \in H^{0}$ given by

$$
\left.p_{h}\right|_{l_{i}}:=g-Q_{h} g-\sum_{k=m+1}^{M} h^{k} P_{k}\left(\frac{\cdot-s_{i}}{h}\right) g^{(k)}, \quad i=1, \ldots, N .
$$

By the Sobolev embedding theorem, the continuity of $V$ and (16), we have that

$$
\max _{\sigma \in \mathbb{R}}\left|V p_{h}(\sigma)\right| \leq C\left\|V p_{h}\right\|_{1} \leq C^{\prime}\left\|p_{h}\right\|_{0}=\mathscr{O}\left(h^{M+1}\right)
$$

and thus

$$
\begin{aligned}
V\left(g-Q_{h} g\right)(\sigma)= & \sum_{k=m+1}^{M} h^{k}\left(\beta_{k}(\sigma ; h)+\sum_{j=1}^{N} \int_{l_{j}} P_{k}\left(\frac{s-s_{j}}{h}\right) B(\sigma, s) g^{(k)}(s) d s\right) \\
& +\mathscr{O}\left(h^{M+1}\right)
\end{aligned}
$$

uniformly in $\sigma \in \mathbf{R}$.

With the change of variable $t=\left(s-s_{j}\right) / h$ and by the Euler-Maclaurin formula it follows that

$$
\begin{aligned}
& \sum_{j=1}^{N} \int_{I_{j}} P_{k}\left(\frac{s-s_{j}}{h}\right) B(\sigma, s) g^{(k)}(s) d s \\
& \quad=\int_{-1 / 2}^{1 / 2} P_{k}(t)\left(h \sum_{j=1}^{N} B\left(\sigma, s_{j}+h t\right) g^{(k)}\left(s_{j}+h t\right)\right) d t \\
& \quad=\alpha_{k} \int_{0}^{1} B(\sigma, s) g^{(k)}(s) d s+\mathscr{O}\left(h^{r}\right)
\end{aligned}
$$

for all $r$, uniformly in $\sigma$. The result is then proven.

We now turn our attention to studying $\beta_{k}(\cdot ; h)-\alpha_{k} V_{0} g^{(k)}$ at the points $s_{i}+\zeta h$. Notice that the kernel of $V_{0}$ is not smooth and the simple arguments leading to (19) do not apply here. 
PROOF OF PROPOSITION 1. Consider first the error functional for the displaced trapezoidal rule for periodic functions

$$
E_{p}[f ; u, h]:=h \sum_{j=1}^{N} f(h(j+u))-\int_{0}^{1} f(x) d x
$$

Let now $c_{k}(\sigma, s):=A(\sigma, s) g^{(k)}(s)$ and $d_{k}(\sigma, s):=c_{k}(\sigma, \sigma+s) \log \left(\sin ^{2}(\pi s)\right)$. By a change of variables

$$
\begin{aligned}
\beta_{k}\left(s_{i}\right. & +\zeta h ; h) \\
& =\int_{-1 / 2}^{1 / 2} P_{k}(t)\left(h \sum_{j=1}^{N} c_{k}\left(s_{i}+\zeta h, s_{j}+t h\right) \log \left(\sin ^{2}\left(\pi\left(s_{j-i}+(t-\zeta) h\right)\right)\right)\right) d t \\
& =\alpha_{k} V_{0} g^{(k)}\left(s_{i}+\zeta h\right)+\int_{-1 / 2}^{1 / 2} P_{k}(t) E_{p}\left[d_{k}\left(s_{i}+\zeta h, \cdot\right), t-\zeta, h\right] d t
\end{aligned}
$$

A straightforward application of Proposition 20 in the Appendix yields

$$
\begin{aligned}
\int_{-1 / 2}^{1 / 2} & P_{k}(t) E_{p}\left[d_{k}(y, \cdot), t-\zeta, h\right] d t \\
= & \sum_{n=0}^{T-1} \frac{1}{n !}\left(c_{k}(y, \cdot)\right)^{(n)}(y) C_{P_{k}, n}(-\zeta) h^{n+1}+O\left(h^{T+1}\right)
\end{aligned}
$$

uniformly for all $y \in \mathbf{R}$. We then obtain the result by combining Proposition 6, (20) and (21). Indeed, if we denote

$$
\begin{aligned}
\mathscr{W}_{k}(\zeta, g)(\sigma) & :=\sum_{j=0}^{k-m-1} C_{P_{k-j}, j}(-\zeta) \frac{1}{j !}\left(A(\sigma, \cdot) g^{(k-j)}\right)^{(j)}(\sigma) \\
& =\sum_{j=m+1}^{k} a_{j, k}(\sigma) b_{j, k}(\zeta) g^{(j)}(\sigma)
\end{aligned}
$$

(the functions $a_{j, k}$ are smooth and periodic), we easily obtain

$$
\begin{aligned}
V\left(g-Q_{h} g\right)\left(s_{i}+\zeta h\right)= & \sum_{k=m+1}^{M} \alpha_{k} h^{k} V g^{(k)}\left(s_{i}+\zeta h\right) \\
& +\sum_{k=m+1}^{M-1} h^{k+1} \mathscr{W}_{k}(\zeta, g)\left(s_{i}+\zeta h\right)+\mathscr{O}_{*}\left(h^{M+1}\right)
\end{aligned}
$$

Notice that $\alpha_{k}=0$ if $k$ is odd, and then the expansion begins at $h^{m+2}$ if $m$ is even. 
REMARK 7. In fact, considering $\zeta=-1 / 2$ if $m$ is odd and $\zeta=0$ if $m$ is even, we have obtained expansions for $V\left(g-Q_{h} g\right)\left(z_{i}\right)$. We remark that by properties of the coefficients $C_{P_{k}, l}(\zeta)$ (notice that $P_{k}$ is even/odd if $k$ is even/odd and applying Lemma 17 in the Appendix), it follows that

$$
\mathscr{W}_{k}(0, \cdot) \equiv \mathbb{W}_{k}(-1 / 2, \cdot) \equiv 0, \quad \text { if } k \text { is odd }
$$

Therefore in (22) the first group of addenda includes only even powers of $h$ (and derivatives of $g$ of even order), whereas the second one includes only odd powers of $h$.

\section{An inf-sup condition}

In this section we prove a uniform infimum-supremum condition from which Proposition 2 follows readily. We will also prove an estimate on the $\|\cdot\|_{-1}$ norm of elements of $T_{h}$.

Let us consider the vector space of periodic polygonal functions

$$
X_{h}:=\left\{u_{h} \in \mathscr{C}^{0}:\left.u_{h}\right|_{\left(z_{i-1}, z_{i}\right)} \in \mathbf{P}_{1}, \forall i\right\}
$$

We remark that the knots of these functions are displaced with respect to the knots of the splines if $\boldsymbol{m}$ is even, since knots are placed on nodes. Let us consider the hat-function basis of $X_{h},\left\{\psi_{1}, \ldots, \psi_{N}\right\}$, where $\psi_{i} \in X_{h}$ is such that $\psi_{i}\left(z_{j}\right)=\delta_{i, j}$ for all $i, j, \delta_{i, j}$ being the Kronecker symbol. The interpolation operator onto $X_{h}$, $Q_{N}: H^{1} \subset \mathscr{C}^{0} \rightarrow X_{h} \subset H^{1}$, is then trivially given by the relation

$$
Q_{N} u=\sum_{i=1}^{N} u\left(z_{i}\right) \psi_{i}=\sum_{i=1}^{N}\left(u, \delta_{i}\right)_{0} \psi_{i}
$$

Notice that the collocation solution to $V g=f$ is the unique function $g_{h} \in V_{h}$ such that $Q_{N} V g_{h}=Q_{N} f$. Consider then the adjoint operator of $Q_{N}, Q_{N}^{*}: H^{-1} \rightarrow H^{-1}$. We therefore have that $\left(Q_{N} u, v\right)_{0}=\left(u, Q_{N}^{*} v\right)_{0}$, for all $u \in H^{1}, v \in H^{-1}$. Then for all $t \in H^{-1}$

$$
Q_{N}^{*} t=\sum_{i=1}^{N}\left(\psi_{i}, t\right)_{0} \delta_{i} \in T_{h}
$$

PROPOSITION 8. There exists $\beta>0$ such that for $h$ small enough

$$
\inf _{0 \neq v_{h} \in v_{h}} \sup _{0 \neq \iota_{h} \in \tau_{h}} \frac{\left|\left(V v_{h}, t_{h}\right)_{0}\right|}{\left\|v_{h}\right\|_{0}\left\|t_{h}\right\|_{-1}} \geq \beta
$$


PROOF. The proof of this result is based on [5]. Given $v_{h} \in V_{h}$, we define $w_{h}:=$ $Q_{N} V v_{h}$. Since $Q_{N}^{2}=Q_{N}$, we have

$$
Q_{N} V v_{h}=w_{h}=Q_{N} w_{h}=Q_{N} V\left(V^{-1} w_{h}\right)
$$

and therefore

$$
v_{h}=C_{h} V^{-1} w_{h} .
$$

By the uniform boundedness of the operators $C_{h}: H^{0} \rightarrow H^{0}$ for $h$ small enough it follows that

$$
\left\|v_{h}\right\|_{0} \leq C\left\|V^{-1} w_{h}\right\|_{0} \leq C^{\prime}\left\|w_{h}\right\|_{1}
$$

Moreover

$$
\begin{aligned}
& \left\|w_{h}\right\|_{1}=\sup _{0 \neq t \in H^{-1}} \frac{\left|\left(w_{h}, t\right)_{0}\right|}{\|t\|_{-1}}=\sup _{\substack{0 \neq t \in H^{-1}\\
}} \frac{\left|\left(V v_{h}, Q_{N}^{*} t\right)_{0}\right|}{\|t\|_{-1}} \\
& =\sup _{\substack{t \in H^{-1} \\
Q_{N}^{*} t \neq 0}}\left(\frac{\left\|Q_{N}^{*} t\right\|_{-1}}{\|t\|_{-1}} \frac{\left|\left(V v_{h}, Q_{N}^{*} t\right)_{0}\right|}{\left\|Q_{N}^{*} t\right\|_{-1}}\right) \leq\left\|Q_{N}^{*}\right\|_{\substack{0 \neq t_{h} \in \tau_{h} \\
0.1}} \frac{\left|\left(V v_{h}, t_{h}\right)_{0}\right|}{\left\|t_{h}\right\|_{-1}},
\end{aligned}
$$

from whence the result follows, since $\left\|Q_{N}^{*}\right\|=\left\|Q_{N}\right\| \leq C$ for all $N$.

LEMMA 9. There exist two positive constants $C_{1}, C_{2}$, independent of $h$, such that for all $t_{h} \in \sum_{i=1}^{N} t_{i} \delta_{i} \in T_{h}$,

$$
C_{1} h \sum_{i=1}^{N}\left|t_{i}\right| \leq\left\|t_{h}\right\|_{-1} \leq C_{2} \sum_{i=1}^{N}\left|t_{i}\right| .
$$

PROOF. By the Sobolev embedding theorem it follows that

and therefore

$$
\left|\left(u, t_{h}\right)_{0}\right| \leq \sum_{i=1}^{N}\left|t_{i}\right|\left|u\left(z_{i}\right)\right| \leq C_{2}\|u\|_{1} \sum_{i=1}^{N}\left|t_{i}\right|
$$

$$
\left\|t_{h}\right\|_{-1}=\sup _{0 \neq u \in H^{1}} \frac{\left|\left(u, t_{h}\right)_{0}\right|}{\|u\|_{1}} \leq C_{2} \sum_{i=1}^{N}\left|t_{i}\right|
$$

Consider now $u_{h}:=\sum_{i=1}^{N} \bar{t}_{i} \psi_{i} \in X_{h}$. Then

$$
\left\|t_{h}\right\|_{-1} \geq \frac{\left|\left(u_{h}, t_{h}\right)_{0}\right|}{\left\|u_{h}\right\|_{1}}=\frac{1}{\left\|u_{h}\right\|_{1}} \sum_{i=1}^{N}\left|t_{i}\right|^{2}
$$

On the other hand

$$
\sum_{i=1}^{N}\left|t_{i}\right| \leq h^{-1 / 2}\left(\sum_{i=1}^{N}\left|t_{i}\right|^{2}\right)^{1 / 2} .
$$


By the explicit polynomial expression of $u_{h}$,

$$
\left\|u_{h}\right\|_{1}^{2}=\left|\int_{0}^{1} u_{h}(s) d s\right|^{2}+\int_{0}^{1}\left|u_{h}^{\prime}(s)\right|^{2} d s \leq 5 h^{-1} \sum_{i=1}^{N}\left|t_{i}\right|^{2}
$$

and hence it follows that

$$
\left\|t_{h}\right\|_{-1} \geq \frac{h}{\sqrt{5}} \sum_{i=1}^{N}\left|t_{i}\right|
$$

\section{The even case}

In this section we consider the particular case of (4) with even data and coefficients, looking for even solutions. That is, consider (4) with $A, B$ smooth 1-periodic and even in both variables, $A$ satisfying (5) and $f$ even. This kind of equation arises from BIE on smooth open arcs with the cosine change of variable (see [19] and Examples 3 and 4 below).

Consider the even periodic Sobolev spaces [20]

$$
H_{e}^{r}:=\left\{u \in H^{r}: \hat{u}(k)=\hat{u}(-k), \quad \forall k\right\} .
$$

Then $H_{e}^{r}$ is a closed subspace of $H^{r}$. The space $H_{e}^{0}$ corresponds to the periodization of functions in $L^{2}(0,1)$ such that $u(1-t)=u(t)$ almost everywhere. Finally, the identification of $H_{e}^{0}$ with its dual can be extended to give a representation of the duality between $H_{e}^{r}$ and $H_{e}^{-r}$ for all $r \in \mathbf{R}$. It is easy to show that $V: H_{e}^{r} \rightarrow H_{e}^{r+1}$ is bounded for all $r \in \mathbf{R}$, if $A$ and $B$ are even. We then assume the following.

HYPOTHESIS 2. The operator $V: H_{e}^{r} \rightarrow H_{e}^{r+1}$ is an isomorphism for all $r \in \mathbf{R}$, or equivalently, its kernel is null for a single and arbitrary value of $r$.

For a discussion on this hypothesis see the comments at the end of this section. Given $f \in H_{e}^{r}$, with $r>1 / 2$, we consider the operator equation,

$$
g \in H_{e}^{r-1}, \quad V g=f
$$

whose solution is going to be approximated by a collocation method.

Consider now the discrete space $V_{h}$ as in Sections 2-5 and define $V_{h, e}:=V_{h} \cap H_{e}^{0}$, its subspace of even splines. In order for this space not to be trivial (equal to $\mathbf{P}_{0}$ ) it is necessary that the origin $x=0$ is either a knot or a midpoint of the grid (that is, $s_{i}-h / 2=0$ or $s_{i}=0$ for some $i$ ). With the choice $s_{0}=0$, the dimension of $V_{h, e}$ is $[N / 2]+1$. For a detailed discussion, restricted to the case when $N$ is even, see [13]. Moreover, if $Q_{h}: \mathscr{C}_{0} \rightarrow V_{h}$ is the interpolation operator defined in Section 2, it can 
be proven by a simple uniqueness argument (see [13]) that if $u \in \mathscr{C}^{\circ}$ is even, then $Q_{h} u \in V_{h, e}$.

We then consider the collocation approximation to (23)

$$
\begin{array}{l|l}
\left(P_{h, e}\right) & \begin{array}{l}
g_{h} \in V_{h, e}, \\
Q_{h} V g_{h}=Q_{h} f=Q_{h} V g,
\end{array}
\end{array}
$$

or equivalently $\operatorname{Vg}\left(z_{i}\right)=f\left(z_{i}\right)$ for $i=1, \ldots, N$ although not all these equations are linearly independent.

The analysis of collocation methods in the even case follows from [19], where the results are just stated for $A(\sigma, s) \equiv A_{0} \in \mathbf{R}$, and in some specific norms. However, with the same proofs plus some standard arguments it can be easily proven that for $h$ small enough, the problem $\left(P_{h, e}\right)$ is uniquely solvable; the even collocation operator $C_{h, e}: H_{e}^{t} \rightarrow V_{h, e}$ is well defined for $t>-1 / 2$ and has boundedness properties identical to those for $C_{h}$; for $-1 \leq s \leq t \leq m+1, s<m+1 / 2, t>-1 / 2$,

$$
\left\|g-C_{h, e} g\right\|_{s} \leq C h^{t-s}\|g\|_{t}, \quad \forall g \in H_{e}^{t} .
$$

For a Petrov-Galerkin formulation like (12) to be given it is necessary to do some simple adaptations. If we consider a Dirac delta distribution $\delta_{x} \in H^{-1}$ such that

$$
\left(\delta_{x}, u\right)_{0}=u(x), \quad \forall u \in H^{1},
$$

then $\left(\delta_{x}+\delta_{-x}\right) / 2 \in H_{e}^{-1}$. This fact together with the symmetry of the nodes $\left\{z_{i}\right\}$ permits us to give a basis of $T_{h, e}:=T_{h} \cap H_{e}^{-1}$. Then $\left(P_{h, e}\right)$ is equivalent to

$$
\mid \begin{aligned}
& g_{h} \in V_{h, e}, \\
& \left(V g_{h}, t_{h}\right)_{0}=\left(f, t_{h}\right)_{0}, \quad \forall t_{h} \in T_{h, e} .
\end{aligned}
$$

Then we can prove a similar result to Proposition 2 for the even case. We can also prove the consistency result.

PROPOSITION 10. Let $g \in \mathscr{C}^{\infty}$ be even. Then there exists a sequence of even functions $\left\{\varepsilon_{k}\right\} \subset \mathscr{C}^{\infty}$ such that for all $M$

$$
V\left(I-Q_{h}\right) g\left(z_{i}\right)=\sum_{k=p(m)}^{M} h^{k} \varepsilon_{k}\left(z_{i}\right)+\mathscr{O}_{*}\left(h^{M+1}\right)
$$

PROOF. We apply Proposition 1 with $\zeta=0$ if $m$ is even and $\zeta=-1 / 2$ if $m$ is odd. We remark that we can have even functions in the expansion by simply noticing that $V\left(I-Q_{h}\right) g$ is even if $g$ is even and then $\varepsilon_{k}(s):=\left(e_{k, \zeta}(s)+e_{k, \zeta}(-s)\right) / 2$ satisfies the same equality in the nodes, since $z_{i}=s_{i}+\zeta h$ is a node if and only if $-z_{i}$ is a node. 
We then can state and prove the theorem corresponding to Theorem $\mathbf{3}$ for the even case without any difficulty.

THEOREM 11. In the hypotheses of this section, Theorem 3 holds for the even collocation operator $C_{h, e}$.

EXAMPLE 3. Let $\Gamma$ be a smooth open arc, given by a parameterization $x:[-1,1] \rightarrow$ $\Gamma \subset \mathbf{R}^{2}$, such that $\left|\mathbf{x}^{\prime}(s)\right|>0$ for all $s$ and $\mathbf{x}(\sigma) \neq \mathbf{x}(s)$ for all $\sigma \neq s$. We are interested in the integral equation

$$
\frac{\imath}{4} \int_{\Gamma} H_{0}^{(1)}(\lambda|\mathbf{y}-\mathbf{z}|) q(\mathbf{z}) d \gamma(\mathbf{z})=u_{0}(\mathbf{y}), \quad \mathbf{y} \in \Gamma
$$

where $H_{0}^{(1)}$ is the Hankel function (see Example 2) and $\lambda \in \mathbf{C}$. The single layer (8) then gives a solution of (6). Now let $\mathbf{a}: \mathbf{R} \rightarrow \Gamma$ be such that

$$
\mathbf{a}(s):=\mathbf{x}(\cos (2 \pi s))
$$

and

$$
g(s):=2 \pi q(\mathbf{a}(s))\left|\mathbf{x}^{\prime}(\cos (2 \pi s))\right||\sin (2 \pi s)|, \quad f(s):=u_{0}(\mathbf{a}(s)) .
$$

Then (25) is equivalent to

$$
\frac{l}{8} \int_{-1 / 2}^{1 / 2} H_{0}^{(1)}(\lambda|\mathbf{a}(\cdot)-\mathbf{a}(s)|) g(s) d s=f .
$$

By (7) and since

$$
\begin{aligned}
\log |\mathbf{a}(\sigma)-\mathbf{a}(s)|^{2} & =C(\sigma, s)+\log |\cos (2 \pi \sigma)-\cos (2 \pi s)|^{2} \\
& =C(\sigma, s)+\log 4+\log \left(\sin ^{2}(\pi(\sigma-s))\right)+\log \left(\sin ^{2}(\pi(\sigma+s))\right),
\end{aligned}
$$

with $C$ smooth and even, the symmetries of the functions involved allow us to write (26) in the form of (4) with even coefficients and data.

EXAMPLE 4. For the Laplace equation (see Example.1) with Dirichlet data on a open arc, the same arguments apply to transform the boundary integral equations into an equation of the form of (4) with $A(\sigma, s) \equiv 1$. This kind of equation has been widely studied from the theoretical and numerical point of view: see [19] and [20].

Instead of the invertibility hypothesis on the even spaces $H_{e}^{r}$, we could assume the more demanding Hypothesis 1 . If $A(\sigma, s) \equiv A_{0}(\sigma)$, both hypotheses can be shown to be equivalent, but this is not the case in the general situation. If Hypothesis 1 holds, then it can be proven that $C_{h}=C_{h, e}$, that is, the collocation solution is even if the data 
and coefficients are even (and the grid respects this symmetry). The analysis given in this section is then trivial, as is in general the analysis of the collocation method under this hypothesis. However, for equations like those arising from the single-layer model for the Helmholtz equation, it is possible that the operator $V$ has a kernel formed by smooth odd functions with no physical basis.

\section{Other aspects}

8.1. Systems All of the preceding analysis can be extended to systems of integral equations with logarithmic principal part coupled with some integral side conditions and with the addition of a finite number of scalar unknowns. We simply state the kind of systems for which the analysis is valid.

We denote by $\mathbf{C}^{p \times q}$ the set of $p \times q$ complex matrices. Let $n$ and $p$ be integers satisfying $n \geq 1, p \geq 0$. Now let $\mathbf{A}, \mathbf{B}: \mathbf{R}^{2} \rightarrow \mathbf{C}^{n \times n}$ be matrix-valued functions, 1 -periodic in both variables and $\mathscr{C}^{\infty}$. Assume also that

$$
\operatorname{det} \mathbf{A}(\sigma, \sigma) \neq 0, \quad \forall \sigma .
$$

Let $\mathbf{M}: \mathbf{R} \rightarrow \mathbf{C}^{n \times p}$ and $\mathbf{L}: \mathbf{R} \rightarrow \mathbf{C}^{p \times n}$ also be smooth and 1-periodic. Finally let $\mathbf{R} \in \mathbf{C}^{p \times p}$. Then we consider the following system of integral equations: given $\mathbf{f} \in\left(H^{r+1}\right)^{n}$ and $\mathbf{b} \in \mathbf{C}^{p}$, find $\mathbf{g} \in\left(H^{r}\right)^{n}$ and $\mathbf{c} \in \mathbf{C}^{p}$ such that

$$
\begin{aligned}
& \int_{0}^{1}\left(\log \left(\sin ^{2}(\pi(\cdot-s))\right) \mathbf{A}(\cdot, s)+\mathbf{B}(\cdot, s)\right) \mathbf{g}(s) d s+\mathbf{M c}=\mathbf{f}, \\
& \int_{0}^{1} \mathbf{L}(s) \mathbf{g}(s) d s+\mathbf{R c}=\mathbf{b},
\end{aligned}
$$

where integration is understood componentwise. Considering the product Sobolev spaces $\mathscr{H}^{r}:=\left(H^{r}\right)^{n} \times \mathrm{C}^{p}$ one can build the matrix operator

$$
\mathscr{V}:=\left(\begin{array}{ll}
V & \mathbf{M} \\
L & \mathbf{R}
\end{array}\right): \mathscr{H}^{r} \rightarrow \mathscr{H}^{r+1}
$$

where $V$ is the integral operator in (27) and $L$ is the functional in (28). If we assume that $\mathscr{V}$ is an isomorphism, then the collocation method associated with the space $\left(V_{h}\right)^{n} \times \mathbf{C}^{p}$ is stable and convergent (see [1]) and we can follow step by step all the analysis given in Sections 3-5. We simply remark that the extension of the interpolation operator $Q_{h}$ to this situation is simply componentwise interpolation for the functional part and the identity for the scalars.

A wide set of examples where systems (27)-(28) appear can be found in [9] and [13]. These include: the Dirichlet problem for the Laplace equation outside a set of nonintersecting smooth closed curves (one could easily adapt the analysis in case some 
of them are open arcs as in Section 6); the first fundamental problem of planar linear elasticity; the Hsiao-MacCamy asymptotic approach to the exterior Stokes problem for viscous fluid flows around a cylindrical object; and a problem in electromagnetism related to the skin-effect taken from [16]. To these we could easily add the Dirichlet problem for the Helmholtz equations in the exterior of a group of smooth curves.

8.2. Potentials In this section we show how the asymptotic expansions of Theorem 3 can be used to prove the existence of asymptotic expansions of the error of the action of some operators on the approximate solution of the BIE. In fact, the aim of solving equations in the form of (4) is often the numerical solution of an associated BVP (see examples above). Once we have solved the BIE we put our solution as a density on the boundary in the representation formula for the solution of the BVP. This gives an exact solution of the differential equation approximately satisfying the boundary conditions.

What we do in these situations is insert the numerical solution into an integral operator

$$
T g(\mathbf{y}):=\int_{0}^{1} F(y, s) g(s) d s,
$$

where $F: \mathbf{R}^{2} \backslash \Gamma \times \mathbf{R} \rightarrow \mathbf{C}$ is smooth and periodic in its second variable. We will restrict our attention to calculation 'far enough' from the boundary, that is, to $F: \Omega \times \mathbf{R} \rightarrow \mathbf{C}$ such that $F \in \mathscr{C}^{\infty}(\bar{\Omega} \times \mathbf{R})$. To avoid the insertion of adequate weights to deal with the behaviour of $F$ at infinity, we will assume that $\Omega$ is a bounded set of $\mathbf{R}^{2}$ (in applications such as those from Examples 1 and $2, \Omega$ is such that $\bar{\Omega} \cap \Gamma=\emptyset$ ).

Then let $X:=\mathscr{C}^{k}(\bar{\Omega})$ for some $k$, endowed with its natural norm. Then $T$ : $H^{-2} \rightarrow X$ is linear and bounded, so it profits from the best convergence estimates of the collocation method: in norm $\|\cdot\|_{-2}$ if $m$ is even and $\|\cdot\|_{-1}$ otherwise.

We introduce the symbol $\mathscr{O}_{X}$ to have the following meaning: when we write $a(h)=\mathscr{O}_{X}\left(h^{k}\right)$, we mean that $\|a(h)\|_{X}=\mathscr{O}\left(h^{k}\right)$. We recall the definition of the parameter $p(m)$ in (10) and of the coefficients $\alpha_{k}$ in (17).

LEMMA 12. Let $f \in \mathscr{C}^{2 M+2}$. Then

$$
T\left(f-Q_{h} f\right)=\sum_{k=p(m) / 2}^{M} \alpha_{2 k} h^{2 k} T f^{(2 k)}+O_{X}\left(h^{2 M+2}\right) .
$$

PROOF. The proof of this result follows from the proof of Theorem 10 in [15]. It can be easily proven by the application of expansions (16) with the same techniques as in the proof of Proposition 6.

We now use this lemma to prove the existence of asymptotic expansions of the potentials calculated by means of the solutions of the collocation method. For the sake of simplicity we state the result for $g$ very smooth. 
THEOREM 13. Let $g \in \mathscr{C}^{\infty}$. Then there exist functions $g_{k} \in X$ such that

$$
T\left(C_{h} g-g\right)=\sum_{k=p(m) / 2}^{p(m)} h^{2 k+1} g_{2 k+1}+\sum_{k=2 p(m)+2}^{M} h^{k} g_{k}+\sigma_{X}\left(h^{M+1}\right) .
$$

PROOF. By the best convergence result we can prove that

$$
T\left(C_{h} g-g\right)=\|g\|_{p(m)} \sigma_{X}\left(h^{p(m)+1}\right) .
$$

Applying this and Theorem 10 in [15] we obtain the expansion

$$
T\left(C_{h} g-g\right)=\sum_{k=p(m)+1}^{M} h^{k} g_{k}+\sigma_{X}\left(h^{M+1}\right) .
$$

We now show that $g_{k}=0$ for $k$ even, $k \leq 2 p(m)$. By (22) and Remark 7, plus the usual boundedness arguments and Proposition 2, we obtain

$$
T\left(C_{h}-Q_{h}\right) g=\sum_{k=p(m) / 2}^{M} \alpha_{2 k} h^{2 k} T C_{h} g^{(2 k)}+\sum_{k=p(m) / 2}^{M} h^{2 k+1} T C_{h} f_{2 k+1}+\sigma_{X}\left(h^{2 M+2}\right),
$$

for some smooth $f_{j}$. Subtracting the expansion in Lemma 12 we obtain

$$
\begin{aligned}
T\left(C_{h} g-g\right)= & \sum_{k=p(m) / 2}^{M} h^{2 k+1} T f_{2 k+1}+\sum_{k=p(m) / 2}^{M} h^{2 k+1} T\left(C_{h} f_{2 k+1}-f_{2 k+1}\right)+ \\
& +\sum_{k=p(m) / 2}^{M} \alpha_{2 k} h^{2 k} T\left(C_{h} g^{(2 k)}-g^{(2 k)}\right)+\sigma_{X}\left(h^{2 M+2}\right) .
\end{aligned}
$$

This expression gives an alternative proof of the fact that $T\left(C_{h} g-g\right)=\sigma_{X}\left(h^{p(m)+1}\right)$ for $g$ smooth enough. Then

$$
T\left(C_{h} g-g\right)=\sum_{k=p(m) / 2}^{p(m)} h^{2 k+1} T f_{2 k+1}+\sigma_{X}\left(h^{2 p(m)+2}\right)
$$

and thus the result is proven.

As an example, Theorem 13 proves that for $m=0$ (collocation with piecewise constant functions)

$$
T C_{h} g=T g+h^{3} g_{3}+h^{5} g_{5}+h^{6} g_{6}+\cdots,
$$

which agrees with a similar result in [12], although there $T$ was a functional, that is, evaluation of the potential was taken in a single point. 
8.3. The Sloan iteration We finish our analysis with a proof that the Sloan iteration for logarithmic equations defined in [8] inherits the existence of an asymptotic expansion of the error. We now restrict the scope of (4) to the case where $A(\sigma, s) \equiv 1$. Consider then the Bessel operator

$$
A g:=\int_{0}^{1} \log \left(4 e^{-1} \sin ^{2}(\pi(\cdot-s))\right) g(s) d s,
$$

which is an isomorphism between $H^{r}$ and $H^{r+1}$ for all $r$ [20]. Moreover, since $A$ is a convolution operator the basic trigonometric polynomials $e^{2 k \pi t}$ are eigenfunctions of $A$.

The equation $V g=f$ is then equivalent to

$$
g+L g=A^{-1} f
$$

where $L:=A^{-1}(V-A): H^{r} \rightarrow H^{s}$ is compact for all $r, s$. The Sloan iteration of the collocation solution to $V g=f$ is then defined as

$$
g_{h}^{*}:=A^{-1} f-L C_{h} g .
$$

Notice that $V-A$ is an integral operator with a smooth kemel.

THEOREM 14. Let $g \in \mathscr{C}^{\infty}$. Then there exist functions $u_{k} \in \mathscr{C}^{\infty}$ such that

$$
g_{k}^{*}-g=\sum_{k=p(m) / 2}^{p(m)} h^{2 k+1} u_{2 k+2}+\sum_{k=2 p(m)+2}^{M} h^{k} u_{k}+\mathscr{O}\left(h^{M+1}\right),
$$

where the equality is valid in $H^{r}$ for all $r$.

PROOF. Notice that $A\left(g_{h}^{*}-g\right)=(V-A)\left(g-C_{h} g\right)$, where

$$
(V-A) g=\int_{0}^{1} F(\cdot, s) g(s) d s,
$$

where $F: \mathbf{R}^{2} \rightarrow \mathbf{R}$ is periodic in both variables and infinitely often differentiable. With very much the same proof as that of Theorem 13, the result follows.

We remark that our result shows the existence of a full asymptotic expansion of the Sloan iterate of the collocation solution, instead of the simpler single-term expansion for the particular case $m=0$ in [8]. The result draws a strong parallelism with the Sloan iterate for the numerical solution of equations of the second kind with smooth kernels, proven in [10]. For practical implementation of the Sloan iterate for logarithmic equations, see [8]. 
8.4. Final comments Once we have obtained asymptotic expansions of the error (pointwise as in Theorem 3 or under the application of some operators as in Theorems 13 or 14) we are allowed to apply polynomial Richardson extrapolation to the limit. This procedure, which can be consulted in any text of basic numerical analysis (for instance, [18]) is of great use for acceleration of convergence and to obtain $a$ posteriori error estimates. The extrapolation thus constitutes a good alternative to using complicated numerical schemes (implementation difficulties increase as $\boldsymbol{m}$ increases), since it combines the advantages of implementing a simple method ( $m=0$ for example) with the higher convergence of more complicated methods.

We do not include numerical experiments, since the collocation method is not a ready-to-implement method without an additional effort of numerical integration. In [2] there is analysis of some possibilities of full discretizations of the collocation method which keep the advantages of giving asymptotic expansions of the error. Some numerical tests are shown there. A full and compact analysis of those full discretizations appears in [3].

\section{Appendix: Euler-Maclaurin formulae for functions with a logarithmic singularity}

Let us consider the error functional for the trapezoidal rule in the whole of $\mathbf{R}$,

$$
E[f ; u, h]:=h \sum_{j=-\infty}^{\infty} f(h(j+u))-\int_{-\infty}^{\infty} f(x) d x
$$

depending 1-periodically on the parameter $u$. As a straightforward consequence of the classical Euler-Maclaurin formula we obtain the following result.

PROPOSITION 15. If $f \in \mathscr{C}^{r}(\mathbf{R})$ is compactly supported, then $E[f ; u, h]=\mathscr{O}\left(h^{r}\right)$ uniformly in $u \in \mathbf{R}$

In what follows we will prove convergence in the mean for the trapezoidal rule for logarithmic-behaved functions. We fix a weight function $\omega \in \mathscr{C}[-1 / 2,1 / 2]$. We denote $\mathscr{D}(\mathbf{R}):=\left\{\varphi \in \mathscr{C}^{\infty}(\mathbf{R}):\right.$ supp $\varphi$ compact $\}$.

LEMMA 16. Let $b_{n}(x):=x^{n} \log x^{2} \varphi(x)$ for $n \geq 0$ and $\varphi \in \mathscr{D}(\mathrm{R})$ such that $\varphi \equiv 1$ in a neighbourhood of $x=0$. Let $\zeta \in[-1 / 2,1 / 2)$. Then there exists $C_{\omega, n}(\zeta)$, independent of $\varphi$, such that for all $r$

$$
\int_{-1 / 2}^{1 / 2} \omega(t) E\left[b_{n} ; t+\zeta, h\right] d t=h^{n+1} C_{\omega, n}(\zeta)+\mathscr{O}\left(h^{r}\right)
$$


Proof. We follow closely the proof of Lemma 6 in [6]. Denote

$$
y(\zeta, h):=\int_{-1 / 2}^{1 / 2} \omega(t) E\left[b_{n} ; t+\zeta, h\right] d t
$$

and notice that $y(\zeta, \cdot) \in \mathscr{C}^{\infty}(0, \infty)$, since for $h>0$, only a finite number of addenda in the definition of $E$ are non-zero. Moreover,

$$
\begin{array}{r}
\frac{\partial y}{\partial h}(\zeta, h)=\frac{1}{h} \int_{-1 / 2}^{1 / 2} \omega(t)\left((n+1) h \sum_{j=-\infty}^{\infty} b_{n}(h(j+t+\zeta))\right. \\
\left.+h \sum_{j=-\infty}^{\infty} \psi(h(j+t+\zeta))\right) d t
\end{array}
$$

where $\psi(x):=x^{n+1} \varphi^{\prime}(x) \log x^{2}+2 x^{n} \varphi(x)$. Since $\psi \in \mathscr{D}(\mathbf{R})$, by Proposition 15 and integration by parts

$$
\begin{aligned}
h \sum_{j=-\infty}^{\infty} \psi(h(j+t+\zeta)) & =\int_{-\infty}^{\infty} \psi(x) d x+\phi\left(h^{r+1}\right) \\
& =-(n+1) \int_{-\infty}^{\infty} b_{n}(x) d x+\sigma\left(h^{r+1}\right),
\end{aligned}
$$

for all $r$, uniformly for all $t$ and $\zeta$. Thus

$$
\frac{\partial y}{\partial h}(\zeta, h)=\frac{n+1}{h} y(\zeta, h)+\mathscr{O}\left(h^{r}\right),
$$

for all $r$, uniformly for all $\zeta$. By the Mean Value Theorem (following exactly the final part of the proof of Lemma 6 in [6]), it holds that $y(\zeta, h)=C_{\omega, n}(\zeta) h^{n+1}+\mathscr{O}\left(h^{r}\right)$, $\zeta$-uniformly.

If we consider $\psi$ to be subject to the same conditions as $\varphi$, then $b_{n}(x)=$ $x^{n} \log x^{2} \psi(x)+f(x)$, with $f \in \mathscr{D}(\mathbf{R})$. By Proposition 15, we get the same expansion as before, and thus $C_{\omega, n}(\zeta)$ does not depend on $\varphi$.

LEMMA 17. If $\omega$ is an even function, then $C_{\omega, n}(-1 / 2)=C_{\omega, n}(0)=0$ for all $n$ odd. Also, if $\omega$ is odd, then $C_{\omega, n}(-1 / 2)=C_{\omega, n}(0)=0$ for $n$ even.

PROOF. In the first case, take $\varphi \in \mathscr{D}(\mathbf{R})$, even and such that $\varphi \equiv 1$ in a neighbourhood of $x=0$. Then by symmetry it follows that

$$
\int_{-1 / 2}^{1 / 2} \omega(t) E\left[b_{n} ; t, h\right] d t=0=\int_{-1 / 2}^{1 / 2} \omega(t) E\left[b_{n} ; t-1 / 2, h\right] d t .
$$

The result then holds. The other case is similar. 
From Lemma 16 and the classical Euler-Maclaurin formula we can obtain asymptotic expansions in the mean for the trapezoidal rule applied to functions of the form $a(x) \log x^{2}$, with $a$ sufficiently smooth and compactly supported.

PROPOSITION 18. Let $a \in \mathscr{C}^{T+1}(\mathbf{R})$ with compact support, and $b(x):=a(x) \log x^{2}$. Then

$$
\int_{-1 / 2}^{1 / 2} \omega(t) E[b ; t+\zeta, h] d t=\sum_{n=0}^{T-2} \frac{a^{(n)}(0)}{n !} C_{\omega, n}(\zeta) h^{n+1}+\mathscr{O}\left(h^{T}\right)
$$

PROOF. By the Taylor theorem and defining $a_{n}:=a^{(n)}(0) / n$ !, we have that

$$
a(x)=\sum_{n=0}^{T} a_{n} x^{n}+r_{T+1}(x) .
$$

Consider then $\varphi \in \mathscr{D}(\mathbf{R})$ such that $\varphi \equiv 1$ in an open set containing supp $a$, so that $b=b \varphi$. Then, by the Euler-Maclaurin formula, $E\left[r_{T+1} \varphi \log \cdot{ }^{2} ; u, h\right]=\mathscr{O}\left(h^{T}\right)$ $u$-uniformly. Thus

$$
\int_{-1 / 2}^{1 / 2} \omega(t) E[b ; t+\zeta, h] d t=\sum_{n=0}^{T} a_{n} \int_{-1 / 2}^{1 / 2} \omega(t) E\left[b_{n} ; t+\zeta, h\right] d t+\varnothing\left(h^{T}\right),
$$

where $b_{n}(x)=x^{n} \log x^{2} \varphi(x)$ as in Lemma 16. Then the result is proven as a simple consequence of that lemma.

Let us now turn our attention to the periodic case. We define the error functional for the trapezoidal rule applied to 1-periodic functions to be

$$
E_{p}[f ; u, h]:=h \sum_{j=1}^{N} f(h(j+u))-\int_{0}^{1} f(x) d x,
$$

depending again 1-periodically on the parameter $u$.

PROPOSIION 19. If $f \in \mathscr{C}^{r}$, then $E_{p}[f ; u, h]=\mathscr{O}\left(h^{r}\right)$ uniformly for all $u$.

PROPOSITION 20. Let $c \in \mathscr{C}^{T+1}, d(x):=c(x) \log \left(\sin ^{2}(\pi x)\right)$ and $\zeta \in[-1 / 2,1 / 2)$. Then

$$
\int_{-1 / 2}^{1 / 2} \omega(t) E_{p}[d ; t+\zeta, h] d t=\sum_{n=0}^{T-2} \frac{c^{(n)}(0)}{n !} C_{\omega, n}(\zeta) h^{n+1}+O\left(h^{T}\right)
$$


PROOF. Consider first an even smooth cut-off function $\varphi \in \mathscr{D}(\mathbf{R})$ such that $\varphi \equiv 1$ in a neighbourhood of $x=0$ and with support included in $(-1 / 2,1 / 2)$. Let $\phi \in \mathscr{C}^{\infty}$ be such that $\varphi+\phi \equiv 1$ in $[-1 / 2,1 / 2]$. Notice the decomposition

$$
d(x)=\phi(x) d(x)+\varphi(x) e(x)+c(x) \log x^{2} \varphi(x),
$$

with

$$
e(x):=c(x) \log \left(\frac{\sin ^{2}(\pi x)}{x^{2}}\right),
$$

where $\phi d \in \mathscr{C}^{T+1}$, whereas $\varphi e \in \mathscr{C}^{T+1}(\mathbf{R})$ has compact support contained in $(-1 / 2,1 / 2)$. By the compactness of the support of $\varphi$ and the periodicity of $d$ and $\phi$, we have

$$
E_{p}[d ; u, h]=E_{p}[\phi d ; u, h]+E[\varphi e ; u, h]+E\left[c \log \cdot{ }^{2} \varphi ; u, h\right] .
$$

By Proposition $19, E_{p}[\phi d ; u, h]=\mathscr{O}\left(h^{T+1}\right)$ uniformly for all $u \in \mathbf{R}$. Likewise $E[\varphi e ; u, h]=\mathscr{O}\left(h^{T+1}\right)$ subject to the same conditions by Proposition 15 .

Then

$$
\int_{-1 / 2}^{1 / 2} \omega(t) E_{p}[d ; t+\zeta, h] d t=\int_{-1 / 2}^{1 / 2} \omega(t) E\left[c \log \cdot{ }^{2} \varphi ; t+\zeta, h\right] d t+\mathscr{O}\left(h^{T+1}\right)
$$

We are now in the hypotheses of Proposition 18 , with $a(x):=c(x) \varphi(x)$. Notice that $a^{(j)}(0)=c^{(j)}(0)$ for all $j$. The result then follows readily.

\section{Acknowledgments}

Both authors were partially supported by DGES Project PB97-1013. The authors would like to thank the referee for suggestions leading to improvements in the paper.

\section{References}

[1] D. N. Arnold and W. L. Wendland, "The convergence of spline collocation for strongly elliptic equations on curves", Numer. Math. 47 (1985) 317-341.

[2] R. Celorrio, "Métodos de contorno para un problema de flujo estacionario alrededor de un túnel", Ph. D. Thesis, Universidad de Zaragoza, Spain, 1997.

[3] R. Celorrio and F.-J. Sayas, "Full collocation methods for some boundary integral equations", Numer. Algorithms 22 (1999) 327-351.

[4] G. Chen and J. Zhou, Boundary element methods (Academic Press, 1992).

[5] M. Costabel, Cours D.E.A. (Université de Rennes I, France, 1992).

[6] M. Crouzeix and F.-J. Sayas, "Asymptotic expansions of the error of spline Galerkin boundary element methods", Numer. Math. 78 (1998) 523-547. 
[7] C. de Boor, A practical guide to splines (Springer, 1978).

[8] I. G. Graham and K. E. Atkinson, "On the Sloan iteration applied to integral equations of the first kind", IMA J. Numer. Anal. 13 (1993) 29-41.

[9] G. C. Hsiao, P. Kopp and W. L. Wendland, "Some applications of a Galerkin-collocation method for boundary integral equations of the first kind", Math. Meth. Appl. Sci. 6 (1984) 280-325.

[10] W. McLean, "Asymptotic error expansions for numerical solutions of integral equations", IMA J. Numer. Anal. 9 (1989) 373-384.

[11] J. Saranen, "The convergence of even degree spline collocation solution for potential problems in smooth domains in the plane", Numer. Math. 53 (1988) 490-512.

[12] J. Saranen, "Extrapolation methods for spline collocation solution of pseudodifferential equations on curves", Numer. Math. 56 (1989) 385-407.

[13] F.-J. Sayas, "The numerical solution of Symm's integral equation on smooth open arcs by spline Galerkin methods", Comp. Math. Appl. 38 (1999) 87-99.

[14] F.-J. Sayas, “Asymptotic expansion of the error of some boundary element methods”, Ph. D. Thesis, Universidad de Zaragoza, Spain, 1994.

[15] F.J. Sayas, “Fully discrete Galerkin methods for systems of boundary integral equations", J. Comp. Appl. Math 81 (1997) 311-331.

[16] H.-Z. Shu, "Approximation d'un problème d'électromagnetisme avec effet de peau", Ph. D. Thesis, Université de Rennes I, France, 1992.

[17] I. H. Sloan and A. Spence, "The Galerkin method for integral equations of the first kind with logarithmic kernel: theory and applications", IMA J. Numer. Anal. 8 (1988) 105-140.

[18] J. Stoer and R. Bulirsch, Introduction to numerical analysis (Springer, 1972).

[19] Y. Yan, "Cosine change of variable for Symm's integral equations on open arcs", IMA J. Numer. Anal. 10 (1990) 521-535.

[20] Y. Yan and I. H. Sloan, "On integral equations of the first kind with logarithmic kernels", J. Int. Eqns Appl. 1 (1988) 549-579. 